\title{
DEGREE FORMULA FOR THE EULER CHARACTERISTIC
}

\author{
OLIVIER HAUTION
}

(Communicated by Lev Borisov)

\begin{abstract}
We give a proof of the degree formula for the Euler characteristic previously obtained by Kirill Zainoulline. The arguments used here are considerably simpler and allow us to remove all restrictions on the characteristic of the base field.
\end{abstract}

\section{INTRODUCTION}

The degree formula for the Euler characteristic says that if $f: Y \rightarrow X$ is a rational map, with $X$ and $Y$ projective connected smooth varieties of the same dimension $d$, then $X$ possesses a zero-cycle of degree

$$
\tau_{d-1} \cdot\left(\chi\left(\mathcal{O}_{Y}\right)-\operatorname{deg} f \cdot \chi\left(\mathcal{O}_{X}\right)\right) .
$$

Here $\operatorname{deg} f$ is the degree of the function fields extension (zero when $f$ is not dominant), $\chi$ is the Euler characteristic, and $\tau_{d-1}$ is the $(d-1)$-st Todd number (the denominator in the $(d-1)$-st Todd class; see (5) $)$. This formula is useful to prove incompressibility properties of some varieties.

In the paper [Zai10, where this formula is introduced, two distinct proofs are given, based on different results:

(a) the generalized degree formula for algebraic cobordism, or

(b) the Rost degree formula.

Both techniques require us to make some assumptions on the characteristic of the base field. It is known that (国) (together with the universal property of algebraic cobordism) implies (b), but (b) has the advantage of being known for some fields of positive characteristic, while (国) requires us to work over a field of characteristic zero.

Indeed $(\underline{b})$ is not proved at the moment when no information on the characteristic of the base field is available, even under the assumption of resolution of singularities. In [Hau, Section 8] we showed that the $p$-primary part of the result of [Zai10] can be obtained in arbitrary characteristic when one disposes of the so-called $p$-resolution of singularities.

The purpose of this article is to give a simpler proof of this result over any field. In contrast to (a) or (b) , the main ingredients here are the Grothendieck-RiemannRoch theorem [Ful98, Theorem 15.2] and a small fraction of [Mer02.

Received by the editors July 10, 2011 and, in revised form, September 15, 2011.

2010 Mathematics Subject Classification. Primary 14C40, 14F43.

Key words and phrases. Grothendieck group, Euler characteristic, degree formula. 


\section{NotATiON}

1.1. Varieties. We fix a base field $k$. A variety is a finite type, separated, quasiprojective scheme over $k$. A morphism of varieties is a morphism of schemes over $k$.

1.2. Grothendieck groups of schemes. (Qui73, $\S 7,1$ and $\S 7,2])$ Let $X$ be a Noetherian scheme. We denote by $K_{0}^{\prime}(X)$ (resp. $\left.K_{0}(X)\right)$ the Grothendieck group (resp. ring) of coherent $\mathcal{O}_{X}$-modules (resp. locally-free $\mathcal{O}_{X}$-modules).

If $f: Y \rightarrow X$ is a flat morphism of Noetherian schemes, then it induces a pullback $f^{*}: K_{0}^{\prime}(X) \rightarrow K_{0}^{\prime}(Y)$.

If $f: Y \rightarrow X$ is a projective morphism of varieties, then it induces a push-forward $f_{*}: K_{0}^{\prime}(Y) \rightarrow K_{0}^{\prime}(X)$.

1.3. Poincaré homomorphism. (Qui73, §7, 1]) There is a natural map

$$
K_{0}(X) \rightarrow K_{0}^{\prime}(X): x \mapsto x \cap\left[\mathcal{O}_{X}\right]
$$

which is an isomorphism when $X$ is regular.

1.4. Rank homomorphism. ([Ful98, Example 15.1.2(d)]) When $X$ is connected, there is a ring homomorphism, sending a vector bundle to its rank

$$
\text { rank: } K_{0}(X) \rightarrow \mathbb{Z} \text {. }
$$

1.5. First Chern class. Let $E$ be a vector bundle over a connected, Noetherian scheme $X$. We denote its first Chern class by

$$
c_{1}(E)=\operatorname{rank} E-\left[E^{\vee}\right] \in K_{0}(X) .
$$

1.6. Subgroup of generically trivial classes. Let $X$ be an integral variety. We denote by $K_{0}^{\prime}(X)^{(1)}$ the subgroup of $K_{0}^{\prime}(X)$ generated by the elements $i_{*}\left[\mathcal{O}_{W}\right]$ with $i$ : $W \hookrightarrow X$ a nondominant closed embedding of varieties. We have an exact sequence

$$
0 \rightarrow K_{0}^{\prime}(X)^{(1)} \rightarrow K_{0}^{\prime}(X) \stackrel{\eta^{*}}{\rightarrow} K_{0}^{\prime}(\operatorname{Spec}(k(X))) \rightarrow 0,
$$

where $\eta: \operatorname{Spec}(k(X)) \rightarrow X$ is the generic point (see Qui73, Proof of $\S 7$, Theorem 5.4, last line p. 131]).

1.7. Euler characteristic. Let $X$ be a projective variety, and $x: X \rightarrow \operatorname{Spec}(k)$ its structural morphism. The Euler characteristic of a coherent $\mathcal{O}_{X}$-module $\mathcal{E}$ is

$$
\chi(\mathcal{E})=\sum_{i \geq 0}(-1)^{i} \operatorname{dim}_{k} H^{i}(X, \mathcal{E})=x_{*}[\mathcal{E}] .
$$

For the last equality we have used the identification $K_{0}^{\prime}(\operatorname{Spec}(k))=\mathbb{Z}$.

\section{Degree formula For $K$-THEORY}

Lemma 2.1. Let $V$ be a vector bundle over a smooth connected variety $X$. Then there exist smooth varieties $Z_{i}$ of dimension $\operatorname{dim} X-1$, projective morphisms $f_{i}: Z_{i}$ $\rightarrow X$, and integers $n_{i}$ such that we have in $K_{0}^{\prime}(X)$,

$$
c_{1}(V) \cap\left[\mathcal{O}_{X}\right]=\sum_{i} n_{i} \cdot\left(f_{i}\right)_{*}\left[\mathcal{O}_{Z_{i}}\right]
$$


Proof. Consider the theory $K=K_{0}(-)\left[t, t^{-1}\right]$ of [Mer02, Example 2.3]. When $f: Y \rightarrow X$ is a projective morphism of smooth connected varieties, then the pushforward $f_{*}^{K}: K(Y) \rightarrow K(X)$ along $f$ is given by the formula, for $x \in K_{0}(Y)$,

$$
f_{*}^{K}\left(x \cdot t^{k}\right)=f_{*}(x) \cdot t^{k+\operatorname{dim} Y-\operatorname{dim} X} .
$$

Here $f_{*}(x) \in K_{0}(X)$ is the element defined by $f_{*}(x) \cap\left[\mathcal{O}_{X}\right]=f_{*}\left(x \cap\left[\mathcal{O}_{Y}\right]\right) \in K_{0}^{\prime}(X)$.

We have in $K(X)$ (see [Mer02, Example 3.1])

$$
c_{1}^{K}(V)=\left(\operatorname{rank} V-\left[V^{\vee}\right]\right) \cdot t^{-1} .
$$

We now use [Mer02, Theorem 9.8], with $A^{*}=K$ as above, and $c^{A}=c_{1}^{K}$. In this theorem $A_{c}^{*}(X)$ is the subgroup of $A^{*}(X)$ generated by projective push-forwards of fundamental classes of smooth varieties (see [Mer02, p. 5]). Merkurjev's theorem says that $c^{A}(V) \cdot 1_{X} \in A_{c}^{*}(X)$; in other words:

$$
c_{1}^{K}(V)=\sum_{i} n_{i} \cdot\left(f_{i}\right)_{*}^{K}\left(1_{Z_{i}}\right),
$$

with $n_{i}, f_{i}, Z_{i}$ as requested. Note that this element belongs to $K^{1}(X)=K_{0}(X) \cdot t^{-1}$; therefore in view of (2) we can choose the varieties $Z_{i}$ so that $\operatorname{dim} Z_{i}=\operatorname{dim} X-1$.

We obtain the result by applying to (3) and (4) the composite

$$
K(X)=K_{0}(X)\left[t, t^{-1}\right] \rightarrow K_{0}(X) \cdot t^{-1}=K_{0}(X) \rightarrow K_{0}^{\prime}(X) .
$$

Proposition 2.2. Let $X$ be a smooth connected variety. Then $K_{0}^{\prime}(X)^{(1)}$ is additively generated by elements of type $f_{*}\left[\mathcal{O}_{Z}\right]$ with $Z$ a smooth variety such that $\operatorname{dim} Z=\operatorname{dim} X-1$, and $f: Z \rightarrow X$ a projective morphism.

Proof. Since $X$ is a regular variety, the map $K_{0}(X) \rightarrow K_{0}^{\prime}(X)$ is an isomorphism. Under this identification, the subgroup $K_{0}^{\prime}(X)^{(1)}$ corresponds to the kernel of the rank homomorphism. Any element $x$ of this kernel can be written as $[E]-[F]$, for some vector bundles $E$ and $F$ on $X$, having the same rank $n$. Then

$$
x=(n-[F])-(n-[E])=c_{1}\left(F^{\vee}\right)-c_{1}\left(E^{\vee}\right) .
$$

Finally we apply Lemma 2.1 above.

Definition 2.3. Let $f: Y \rightarrow X$ be a projective morphism of varieties, with $X$ integral. Consider the generic fiber $Y \times_{X} k(X)$ as a variety over $k(X)$, and define an integer

$$
\operatorname{deg} f=\chi\left(\mathcal{O}_{Y \times_{X} k(X)}\right)
$$

it being understood that $\chi(\emptyset)=0$.

Lemma 2.4. Let $f: Y \rightarrow X$ be a projective morphism, with $X$ integral. Then

$$
f_{*}\left[\mathcal{O}_{Y}\right]-\operatorname{deg} f \cdot\left[\mathcal{O}_{X}\right] \in K_{0}^{\prime}(X)^{(1)} .
$$

Proof. This follows from the sequence (1), and from the commutative diagram

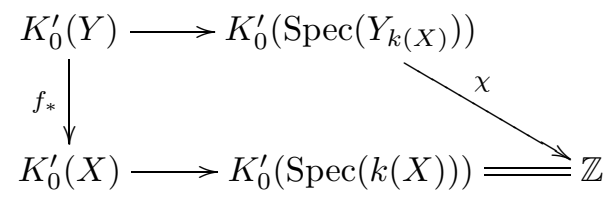


Theorem 2.5. Let $f: Y \rightarrow X$ be a projective morphism, with $X$ a smooth connected variety. Then we have in $K_{0}^{\prime}(X)$,

$$
f_{*}\left[\mathcal{O}_{Y}\right]=\operatorname{deg} f \cdot\left[\mathcal{O}_{X}\right]+\sum_{i} n_{i} \cdot\left(f_{i}\right)_{*}\left[\mathcal{O}_{Z_{i}}\right],
$$

for some smooth varieties $Z_{i}$ of dimension $\operatorname{dim} X-1$, projective morphisms $f_{i}: Z_{i} \rightarrow$ $X$, and integers $n_{i}$.

Proof. This results from the combination of Lemma 2.4 and Proposition 2.2

\section{The Euler CHARACTERISTIC}

Given a rational number $\alpha$, let us denote by $[\alpha]$ the greatest integer $\leq \alpha$. If $d \geq 0$ is an integer, the $d$-th Todd number is

$$
\tau_{d}=\prod_{p \text { prime }} p^{[d /(p-1)]}
$$

These numbers appear as denominators in the Todd class. We have $\tau_{d} \mid \tau_{e}$ whenever $d \leq e$.

The next lemma is an immediate consequence of the Grothendieck-RiemannRoch theorem.

Lemma 3.1 ([Zai10, Lemma 3.6]). If $X$ is a smooth projective variety, then it possesses a zero-cycle of degree $\tau_{\operatorname{dim} X} \cdot \chi\left(\mathcal{O}_{X}\right)$.

If $X$ is a projective variety, we denote by $n_{X}$ the positive integer such that

$$
\operatorname{deg} \mathrm{CH}(X)=n_{X} \cdot \mathbb{Z} .
$$

This integer coincides with the greatest common divisor of the degrees of closed points of $X$.

Proposition 3.2. Let $f: Y \rightarrow X$ be a projective morphism. Assume that $X$ is smooth, projective and connected. Then we have, using Definition 2.3 ,

$$
\tau_{\operatorname{dim} X-1} \cdot \chi\left(\mathcal{O}_{Y}\right)=\operatorname{deg} f \cdot \tau_{\operatorname{dim} X-1} \cdot \chi\left(\mathcal{O}_{X}\right) \bmod n_{X} .
$$

Proof. Project the formula of Theorem 2.5 to $K_{0}^{\prime}(\operatorname{Spec}(k))=\mathbb{Z}$. This gives

$$
\chi\left(\mathcal{O}_{Y}\right)=\operatorname{deg} f \cdot \chi\left(\mathcal{O}_{X}\right)+\sum_{i} n_{i} \cdot \chi\left(\mathcal{O}_{Z_{i}}\right) .
$$

Note that $n_{X} \mid n_{Z_{i}}$. Since every $Z_{i}$ is smooth and of dimension $<\operatorname{dim} X$, Lemma 3.1 gives the result.

Let $X, Y$ be projective integral varieties. A correspondence $Y \rightsquigarrow X$ is an element $\gamma \in \mathrm{CH}(Y \times X)$. The multiplicity mult $\gamma$ is the image of $\gamma$ under the map

$$
\mathrm{CH}(Y \times X) \rightarrow \mathrm{CH}\left(k(Y) \times_{k} X\right) \rightarrow \mathrm{CH}(k(Y))=\mathbb{Z} .
$$

The transpose ${ }^{t} \gamma$ of $\gamma$ is the correspondence $X \rightsquigarrow Y$ corresponding to the image of $\gamma$ under the morphism exchanging factors.

When $\gamma=[\Gamma]$ for some integral closed subvariety $\Gamma$ of $Y \times X$, then mult $\gamma$ can be nonzero only if $\operatorname{dim} \Gamma=\operatorname{dim} Y$. In this case, mult $\gamma$ coincides with the integer $\operatorname{deg}(\Gamma \rightarrow Y)$ of Definition 2.3 . 
Lemma 3.3. Let $\gamma: Y \rightsquigarrow X$ be a correspondence between projective integral varieties, with $Y$ smooth. Then $n_{X} \mid \operatorname{mult} \gamma \cdot n_{Y}$.

Proof. The map mult: $\mathrm{CH}(Y \times X) \rightarrow \mathbb{Z}$ is linear and vanishes on cycles of dimension $\neq \operatorname{dim} Y$; hence we can assume that $\gamma=i_{*}[\Gamma]$, where $i: \Gamma \hookrightarrow Y \times X$ is an integral closed subvariety of dimension $\operatorname{dim} Y$. We have a diagram

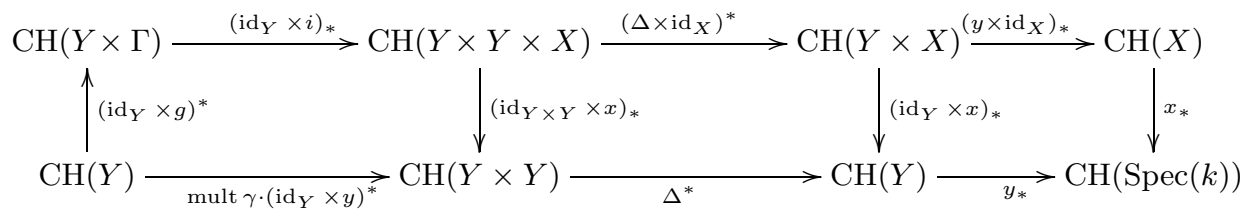

where $\Delta: Y \hookrightarrow Y \times Y$ is the diagonal embedding, and $x: X \rightarrow \operatorname{Spec}(k), y: Y \rightarrow$ $\operatorname{Spec}(k), g: \Gamma \rightarrow \operatorname{Spec}(k)$ the structural morphisms. The square on the left is commutative because the push-forward along the projective morphism $\Gamma \rightarrow Y$ sends $[\Gamma]$ to mult $\gamma \cdot[Y]$. Commutativity of the other squares is clear. We see that the bottom composite is mult $\gamma \cdot y_{*}$ and factors through $x_{*}$.

Theorem 3.4. Let $\gamma: Y \rightsquigarrow X$ be a correspondence between smooth, projective, connected varieties of the same dimension $d$. Then we have

$$
\text { mult } \gamma \cdot \tau_{d-1} \cdot \chi\left(\mathcal{O}_{Y}\right)=\operatorname{mult}^{t} \gamma \cdot \tau_{d-1} \cdot \chi\left(\mathcal{O}_{X}\right) \quad \bmod \operatorname{gcd}\left(n_{Y}, n_{X}\right)
$$

Proof. We can assume as above that $\gamma$ is represented by an integral $d$-dimensional closed subvariety $\Gamma$ of $Y \times X$. The result then follows from the application of Proposition 3.2 to the projective morphisms $\Gamma \rightarrow X$ and $\Gamma \rightarrow Y$.

Let $f: Y \rightarrow X$ be a rational map of integral projective varieties. The closure of its graph defines a correspondence $\gamma_{f}$ of multiplicity one. We define the integer $\operatorname{deg} f$ as mult ${ }^{t} \gamma_{f}$. This is compatible with Definition 2.3.

Combining Theorem 3.4 and Lemma 3.3, we obtain the following generalization of [Zai10, Theorem 5.7].

Corollary 3.5. Let $f: Y \rightarrow X$ be a rational map of projective, smooth, connected varieties of the same dimension $d$. Then we have

$$
\tau_{d-1} \cdot \chi\left(\mathcal{O}_{Y}\right)=\operatorname{deg} f \cdot \tau_{d-1} \cdot \chi\left(\mathcal{O}_{X}\right) \quad \bmod n_{X} .
$$

\section{Remarks AND CONSEQUenCES}

4.1. Incompressibility. Using Corollary 3.5, one can generalize the statements of [Zai10, $6.2, \ldots, 6.6]$ to base fields of arbitrary characteristic.

4.2. Resolution of singularities. When resolution of singularities is available, one can obtain Proposition 2.2 or Lemma 3.1, and therefore Proposition 3.2 for singular $X$. One can thus remove the smoothness assumptions in Theorem 3.4.

When the dimension of $X$ is $<p(p-1)$, where $p$ is the characteristic of the base field, one can also use Hau11 to obtain the same result over any field. 
4.3. Perfect fields of positive characteristic. A statement of Rost ([Ros08, Corollary 1]) says that for any projective variety $X$ over a perfect field of positive characteristic $p$, one has

$$
v_{p}\left(n_{X}\right) \leq v_{p}\left(\chi\left(\mathcal{O}_{X}\right)\right) .
$$

It follows that the $p$-primary content of Theorem 3.4 is empty when the base field is perfect.

4.4. Generalized degree formula for periodic multiplicative theories. By [LM07, Theorem 4.2.10], we know that $K$-theory is the universal oriented weak cohomology theory with periodic multiplicative formal group law. Therefore Theorem 2.5 implies

Proposition 4.1. Let $A$ be an oriented weak cohomology theory (LM07, Definition 4.1.13]) over an arbitrary field $k$. Assume that the formal group law of $A$ is $F(x, y)=x+y-\alpha \cdot x y$, for some invertible element $\alpha \in A(\operatorname{Spec}(k))$. Then for any projective morphism of smooth connected varieties $f: Y \rightarrow X$, we have in $A(X)$,

$$
\alpha^{-\operatorname{dim} Y} \cdot f_{*}\left(1_{Y}\right)=\operatorname{deg} f \cdot \alpha^{-\operatorname{dim} X} \cdot 1_{X}+\sum_{i} n_{i} \cdot \alpha^{1-\operatorname{dim} X} \cdot\left(f_{i}\right)_{*}\left(1_{Z_{i}}\right)
$$

for some smooth varieties $Z_{i}$ of dimension $\operatorname{dim} X-1$, projective morphisms $f_{i}: Z_{i} \rightarrow$ $X$, and integers $n_{i}$.

In particular we obtain that the subgroup of $A(X)$ generated by projective pushforwards of fundamental classes of smooth varieties of arbitrary dimensions ("the image of cobordism") coincides with the $\mathbb{Z}[\alpha]$-submodule of $A(X)$ generated by $1_{X}$ and the projective push-forwards of fundamental classes of smooth varieties of dimensions $<\operatorname{dim} X$.

4.5. Generalized degree formula for connective $K$-theory. Connective $K$ theory $\mathrm{CK}_{p, q}$ has been introduced, over any field, in [Cai08. It appears that the degree formula for this theory is equivalent to the degree formula for $K$-theory.

In order to make a precise statement, we use the notation of Cai08. In addition, for an integral variety $X$, we denote by $[X]$ the element $\left[\mathcal{O}_{X}\right]$ considered as an element of $\mathrm{CK}_{\operatorname{dim} X,-\operatorname{dim} X}(X)$. Thus the Bott element $\beta \in \mathrm{CK}_{1,-1}(\operatorname{Spec}(k))$ is $p_{*}\left[\mathbb{P}^{1}\right]$, where $p: \mathbb{P}^{1} \rightarrow \operatorname{Spec}(k)$ is the projective line.

Proposition 4.2. Let $f: Y \rightarrow X$ be a projective morphism of integral varieties, with $X$ smooth. Assume that $c=\operatorname{dim} X-\operatorname{dim} Y$ is $\leq 0$. Then we have in $\mathrm{CK}_{\operatorname{dim} Y,-\operatorname{dim} Y}(X)$,

$$
f_{*}[Y]=\operatorname{deg} f \cdot \beta^{-c} \cdot[X]+\sum_{i} n_{i} \cdot \beta^{1-c} \cdot\left(f_{i}\right)_{*}\left[Z_{i}\right]
$$

for some smooth varieties $Z_{i}$ of dimension $\operatorname{dim} X-1$, projective morphisms $f_{i}: Z_{i} \rightarrow$ $X$, and integers $n_{i}$.

Proof. It follows from the construction of CK-groups, and from the inequality $\operatorname{dim} Y \geq \operatorname{dim} X$, that the natural map $\mathrm{CK}_{\operatorname{dim} Y,-\operatorname{dim} Y}(X) \rightarrow K_{0}^{\prime}(X)$ is an isomorphism. This maps sends the formula of the proposition to the formula of Theorem 2.5 


\section{ACKNOWLEDGEMENTS}

The support of EPSRC Responsive Mode grant EP/G032556/1 is gratefully acknowledged. The author thanks Alexander Vishik for useful discussions on the subject of this paper.

\section{REFERENCES}

[Cai08] Shuang Cai. Algebraic connective $K$-theory and the niveau filtration. J. Pure Appl. Algebra, 212(7):1695-1715, 2008. MR2400737 (2009a:14003)

[Ful98] William Fulton. Intersection theory, volume 2 of Ergebnisse der Mathematik und ihrer Grenzgebiete. 3. Folge. A Series of Modern Surveys in Mathematics [Results in Mathematics and Related Areas. 3rd Series. A Series of Modern Surveys in Mathematics]. Springer-Verlag, Berlin, second edition, 1998. MR.1644323 (99d:14003)

[Hau] Olivier Haution. Reduced Steenrod operations and resolution of singularities. $J$. K-Theory, 9(2):269-290, 2012. MR2922390

[Hau11] Olivier Haution. Integrality of the Chern character in small codimension. Adv. Math., 231(2):855-878, 2012. MR2955195

[LM07] Marc Levine and Fabien Morel. Algebraic cobordism. Springer Monographs in Mathematics. Springer, Berlin, 2007. MR2286826 (2008a:14029)

[Mer02] Alexander Merkurjev. Algebraic oriented cohomology theories. In Algebraic number theory and algebraic geometry, volume 300 of Contemp. Math., pages 171-193. Amer. Math. Soc., Providence, RI, 2002. MR 1936372 (2003j:14023)

[Qui73] Daniel Quillen. Higher algebraic K-theory. I. In Algebraic K-theory, I: Higher Ktheories (Proc. Conf., Battelle Memorial Inst., Seattle, Wash., 1972), pages 85-147. Lecture Notes in Math., Vol. 341. Springer, Berlin, 1973. MR.0338129 (49:2895)

[Ros08] Markus Rost. On Frobenius, K-theory, and characteristic numbers. Preprint, 2008. http://www.math.uni-bielefeld.de/ ${ }^{\sim}$ rost/frobenius.html.

[Zai10] Kirill Zainoulline. Degree formula for connective K-theory. Invent. Math., 179(3):507522, 2010. MR2587339(2011d:19012)

School of Mathematical Sciences, University of Nottingham, University Park, NotTINGHAM, NG7 2RD, UNITED KINGDOM

E-mail address: olivier.haution@gmail.com 